\title{
Synchronic Modal Equivalencing (SME) for Structure-Preserving Dynamic Equivalents
}

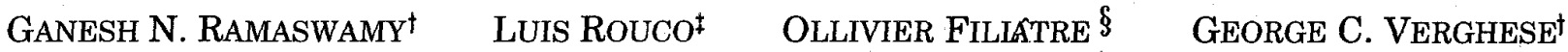

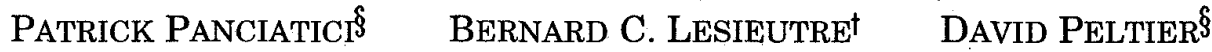 \\ ${ }^{\dagger}$ Massachusetts Institute of Technology $\quad$ †Instituto de Investigación Tecnológica, Madrid $\quad \S_{\text {Electricité de France }}$
}

\begin{abstract}
A novel framework for dynamic equivalencing that we recently introduced in the context of classical swing-equation models is extended in this paper to detailed models in structure-preserving, differential/algebraic-equation form. The system is partitioned into a study area and one or more external areas on the basis of synchrony, a generalization of slowcoherency that forms one leg of our framework. Retaining a detailed model for a single reference generator from each external area, the dynamics of the remaining external generators are then modally equivalenced in the style of Selective Modal Analysis; this modal equivalencing is the other leg of our framework. The equivalenced external generators are thereby collectively replaced by a linear multi-port "admittance", which is easily represented using controlled current injectors at the buses of the replaced generators. The rest of the system model can be retained in its original nonlinear dynamic form. The approach is tested - with encouraging results - on the familiar 93rd-order, 10-machine, 39-bus New England model, using an implementation in the EUROSTAG simulation package.
\end{abstract}

Key Words - Dynamic Equivalents, DifferentialAlgebraic Equations, Synchrony, Selective Modal Analysis, Slow Coherency.

\section{INTRODUCTION AND OVERVIEW}

Constructing reduced-order dynamic models, or dynamic equivalents, is an important challenge in the study of large interconnected power systems, and has been addressed in many papers. An early reference is [20] (but involving modal analysis in a very different way than in the present paper). The coherency-based approach in [9], wirn the more analytical adjuncts in [3], [23], and with the implementation in the DYNRED package [10], constitutes the most important systematic route to dynamic equivalencing in current practice. Recent papers such as

95 WM 113-1 PWRS A paper recommended and approved by the IEEE Power System Engineering Committee of the IEEE Power Engineering Society for presentation at the 1995 IEEE/PES Winter Meeting, January 29, to February 2, 1995, New York, NY. Manuscript submitted August 1, 1994; made available for printing January 3, 1995.
[8], [6], [19], [21], [5] reflect continuing interest in further development of this subject.

In [14] we proposed a new framework for dynamic equivalencing, termed Synchronic Modal Equivalencing (SME), and presented algorithms for constructing effective dynamic equivalents for classical swing-equation models. The present paper generalizes the framework to obtain dynamic equivalents for detailed models described in structure-preserving, differential/algebraic-equation (DAE) form. A structure-preserving DAE description comprises separate specifications of the differentialequation models (in state-space form) for the individual dynamic subsystems and of the sparse algebraic constraints imposed on the subsystems by the interconnecting network. This is the standard way in which power systems are described for time-domain simulation of dynamic behavior, hence the importance of developing dynamic equivalencing directly for DAE descriptions.

Our approach grows out of slow-coherency [3] and multi-area Selective Modal Analysis (SMA) [11], [16]. We first define a study area and external areas using a generalization of slow-coherency termed synchrony in [13], [15]. The area definition procedure involves computations with a classical, linearized, undamped, swing-equation model, and is summarized in Section II.A.

Now, retaining a detailed model for a single reference generator from each external area, the dynamics of the remaining external generators are modally equivalenced in the style of SMA, using modal data from a detailed linearized model. The underlying idea is briefly described in Section II.B in the context of a state-space model of the power system, while Section III presents a more detailed development for DAE models. Through the modal equivalencing step, the equivalenced external generators are collectively replaced by a linear multi-port "admittance", which is easily represented in the DAE description using controlled current injectors at the buses of the replaced generators. The rest of the DAE model can be retained in its original nonlinear dynamic form.

The proposed methodology has been tested on the familiar 93rd-order, 10-machine, 39-bus New England model. Results using linearized models are in Section IV.A, and results from a nonlinear implementation on the EU-

†Address for correspondence: Room 10-050, Laboratory for Electromagnetic and Electronic Systems, Massachusetts Institute of Technology, Cambridge, MA 02139. 
ROSTAG [18], [1] simulation package are in Section IV.B.

\section{BACKGROUND}

\section{A. Determining Synchronic Areas}

We begin our dynamic equivalencing process by decomposing the model into synchronic areas. The underlying idea is similar to slow-coherent decomposition, [3], but synchrony is rather more flexible, and this can give it an advantage over slow-coherency in dealing with practical cases. The restrictive nature of slow-coherency has been noted before and relaxations have been suggested [4], [7].

Synchrony is defined with respect to a selected subset of modes of a linearized model. We call such a subset a chord. Two generators are termed exactly or approximately synchronic in a chord $\nu$, or are $\nu$-synchronic, if their angular variations are exactly or approximately in constant proportion for any transient in which only the modes in $\nu$ are excited. In contrast, $\nu$-coherency requires exact or approximate equality of the angular variations, and slow-coherency further restricts $\nu$ to contain only the slowest electromechanical modes of the system. (The tolerance-based coherency procedure in [4] actually relaxes slow-coherency in the direction of slow-synchrony, although the dynamical implications are not explored.)

A (maximal) set of $\nu$-synchronic generators constitutes a synchronic group, or a synchronic "area". We use quotes to emphasize that a $\nu$-synchronic area need not comprise contiguous generators of the network. However, for the chords that we typically choose, the synchronic areas do tend to have a natural geographic identity.

A simple but generally effective basis for area determination is the classical, linearized, undamped, swingequation model for small-signal angular perturbations of the generators from nominal. The area structure determined from this simple model of electromechanical behavior can then be imposed on a detailed model, because such swing-equation models have been found to preserve quite well the frequencies and mode shapes of the electromechanical modes in detailed models, see [12], [16], [15] (but also [21] for an exception). We briefly describe the swing-equation model next.

Denote by $M_{i}$ the inertia of the $i$ th generator, by $\Delta \delta_{i}$ the deviation of its rotor angle from nominal, and by $\mathcal{J}$ the $n \times n$ matrix whose elements are the synchronizing coefficients. Then the linearized, undamped, swing-equation model is

$$
\Delta \ddot{\delta}=-\mathcal{M}^{-1} \mathcal{J} \Delta \delta
$$

where $\mathcal{M}=\operatorname{Diag}\left[M_{1} \cdots M_{n}\right]$ and $\Delta \delta=\left[\Delta \delta_{1} \cdots \Delta \delta_{n}\right]^{T}$. (Throughout this paper, we drop the time argument $t$ for notational simplicity wherever possible.) The eigenvalues of $-\mathcal{M}^{-1} \mathcal{J}$ are $\sigma_{n-1}<\cdots<\sigma_{1}<\sigma_{0}=0$, each corresponding to a pair of natural frequencies $\left\{\bar{\lambda}_{2 i}=j \sqrt{-\sigma_{i}}, \bar{\lambda}_{2 i+1}=\right.$ $\left.-j \sqrt{-\sigma_{i}}\right\}$ of the system (1).

With the desired number of areas specified by the user, the first step in area determination is to select the chord $\nu$. The slow-coherency approach always picks the slowest electromechanical modes of the system, starting with $\sigma_{0}$. Underlying this choice is a model of the power system as consisting of disjoint areas that are strongly connected internally but coupled to each other by vanishingly weak ties. The electromechanical modes of the system then have a clear slow/fast separation. The slow modes are inter-area modes, and the generators are coherent with respect to these slow modes. The fast modes are intraarea modes.

To deal with models that do not have the ideal slowcoherency structure, we have developed a novel sequential approach to determining good candidate chords, see [13],[15]. The resulting chords are typically not the slowest modes. The importance of such non-standard chords for area determination has been illustrated in applications to multi-area eigenanalysis [15] and dynamic equivalencing [14], and will again become evident in Section IV.

Once the chord has been selected, the (approximately) synchronic groups or areas can be determined through computations on the associated eigenvectors of the matrix $-\mathcal{M}^{-1} \mathcal{F}$ in (1). Details may be found in [13], [15]. The synchrony-based grouping algorithms presented in those papers first determine a set of reference generators corresponding to the chosen chord, using a certain clustering procedure. Each non-reference generator is then assigned to whichever reference generator it has the highest degree of synchrony with. This completes the area determination. Proper area determination plays a key role in our equivalencing approach, as the numerical results in Section IV will confirm. The reasons for its importance are tied to the properties that we mention next.

An aggregate model comprising just the reference generators of the different synchronic areas is able to reproduce (to reasonable accuracy) all the modes in $\nu$, [13], [15]. This decomposability, [3], is a consequence of our chord selection procedure, and justifies labeling the modes in $\nu$ as inter-area modes. The analogous feature in the slowcoherency framework is the fact that the slow modes are inter-area modes, which is a direct consequence of the weak-interconnections model.

It is more surprising that the modes of (1) not in $\nu$ typically behave as intra-area modes for the synchronic grouping, in the sense that each of them is well reproduced by some natural "local" model, i.e. a model comprising all the generators in one synchronic area, [13], [15]. The analogous (multi-) localizability result in the slow-coherency theory, [3], is that the fast modes are intra-area modes, a natural consequence of the weakinterconnections model. Our chord selection procedure can be shown to permit any localizability present in the synchronic groupings to emerge, but we do not yet have an adequate understanding of the conditions under which localizability exists.

\section{B. Constructing a Dynamic Equivalent}

One of the synchronic areas defined through the above procedure is now selected as the study area, based on its inclusion of generators that are of interest in the studies being contemplated. All the other synchronic areas are designated as external areas. Typical dynamic equivalencing approaches involve retaining a full model for the study area and then constructing equivalents for the ex- 
ternal areas. Our approach differs in this respect, as we also retain a detailed description for the reference generator of each external area. This is because, as noted earlier, an aggregate model that includes the reference generators can preserve the inter-area modes. Proper representation of the inter-area behavior is important, because disturbances in the study area can excite the inter-area modes.

The inter-area modes (the modes corresponding to those in $\nu$ ), as well as the intra-area modes local to the study area, will be termed the relevant modes of the original system. The generators that are to be retained in full detail - the reference generators of the external areas and all the generators in the study area - will be called the relevant generators for equivalencing, and their state variables will be termed the relevant state variables. The dynamic models of all other (less relevant) generators will be fully reduced, i.e. replaced by purely algebraic equations. The resulting model will be made to exactly retain a subset (or perhaps all) of the relevant modes; we shall refer to these exactly retained modes as equivalencing modes. Note that our approach does not involve or permit arbitrary definitions of study area boundaries; rather, it indicates which generators can be safely equivalenced for the studies that are planned.

For clarity, we defer to the next section our treatment of a DAE description. Instead, in this subsection we use the following linearized, detailed state-space model to present some of the objectives and issues in SME:

$$
\left[\begin{array}{l}
\dot{\mathbf{x}}_{r} \\
\dot{\mathbf{x}}_{z}
\end{array}\right]=\left[\begin{array}{ll}
\mathcal{A}_{r r} & \mathcal{A}_{r z} \\
\mathcal{A}_{z r} & \mathcal{A}_{z z}
\end{array}\right]\left[\begin{array}{c}
\mathbf{x}_{r} \\
\mathbf{x}_{z}
\end{array}\right]+\left[\begin{array}{c}
\mathcal{B}_{r} \\
\mathbf{0}
\end{array}\right] \mathbf{w}
$$

The subscripts $r$ and $z$ respectively refer to the relevant generators and the remaining (less relevant) generators, and $\mathbf{x}_{r}, \mathbf{x}_{\boldsymbol{z}}$ denote the corresponding state vectors. The external inputs $w$ are shown as being directly applied only to the relevant generators. All the variables in this linearized model represent small-signal perturbations from nominal operation. We denote the natural frequencies of the system in (2), i.e. the eigenvalues of the matrix $\mathcal{A}$ there, by $\left\{\lambda_{i}\right\}$. Of these, there are $2 n-$ the "electromechanical" natural frequencies - that are well approximated by the eigenvalues of (1).

In this state-space setting, our objective is to find a reduced-order model (or dynamic equivalent) of the form

$$
\dot{\mathbf{x}}_{r}=\hat{\mathcal{A}} \mathbf{x}_{r}+\hat{\mathcal{B}} \mathbf{w}
$$

in which the behavior of the state vector $x_{r}$ is approximately the same as in the full system, for the same initial conditions $\mathbf{x}_{r}(0)$ and inputs $\mathbf{w}$. Our approach is to look for a relationship of the form

$$
\mathbf{x}_{x}=\mathbf{K} \mathbf{x}_{\boldsymbol{r}}
$$

that holds approximately in (2), when only relevant modes are excited. We find this $\mathbf{K}$ by solving the equation

$$
\mathbf{V}_{z}=\mathbf{K} \mathbf{V}_{r}
$$

where the $i$ th columns of $\mathbf{V}_{z}$ and $\mathbf{V}_{r}$ respectively are $\mathbf{v}_{z i}$ and $\mathbf{v}_{r i}$, the portions of the eigenvector of $\lambda_{i}$ that correspond to the $z$ and $r$ variables in (2). The index $i$ ranges over the relevant modes, or over some subset of these; the modes that it ranges over constitute the equivalencing modes referred to earlier. Note that the system (5) is under-determined in general, even if all the relevant modes are picked as equivalencing modes, so other considerations have to enter the choice of some particular solution. (There is a unique solution if the external reference generators are all modeled by just swing equations and if all the relevant modes are selected as equivalencing modes.) Now making the substitution (4) in (2), we get a model of the form (3), with

$$
\hat{\mathcal{A}}=\mathcal{A}_{r \boldsymbol{r}}+\mathcal{A}_{\boldsymbol{r} z} \mathbf{K}, \quad \hat{\mathcal{B}}=\mathcal{B}_{\boldsymbol{r}}
$$

Unfortunately, knowledge of $\mathbf{K}$ does not provide an equivalent that can be used in a typical power system (transient stability) simulation package. The power system is not described in state-space form in such a package, and $\mathbf{K}$ does not correlate directly with any structural features of the power system. We therefore turn attention to constructing an equivalent in the structure-preserving DAE setting.

\section{EQUIVALENCING DAE MODELS}

To derive the linearized DAE model that we shall work with, first write the detailed linearized state-space model for the subsystem at the $j$ th bus as

$$
\begin{aligned}
& \dot{\mathbf{x}}_{j}=\mathbf{A}_{j} \mathbf{x}_{j}+\mathbf{B}_{j} \mathbf{u}_{j}+\mathcal{B}_{j} \mathbf{w}_{j} \\
& \mathbf{y}_{j}=\mathbf{C}_{j} \mathbf{x}_{j}+\mathbf{D}_{j} \mathbf{u}_{j}+\mathcal{D}_{j} \mathbf{w}_{j}
\end{aligned}
$$

where $\mathbf{x}_{\boldsymbol{j}}$ is the state vector, $\mathbf{w}_{\boldsymbol{j}}$ is the perturbation in the external input to the subsystem, $\mathbf{u}_{j}$ is the perturbation in the bus voltage phasor, and $\mathbf{y}_{j}$ is the perturbation in the phasor of the current injected into the network at the bus. In this paper we shall treat all loads as static (nondynamic), so their models will have no state equation (7), only an equation of the form (8) with the state-dependent term absent. The algebraic constraints on the network variables are given by

$$
\mathbf{y}=\mathbf{Y u}
$$

where $\mathbf{Y}$ is the (sparse) network admittance matrix, and $\mathbf{u}$ and $\mathbf{y}$ are vectors containing the $\mathbf{u}_{j}$ and $\mathbf{y}_{j}$.

We shall assume that $\mathcal{B}_{j}=0$ for all non-reference, external (i.e. all less relevant) generators. With this assumption, the external inputs $\mathbf{w}_{j}$ do not play a role in the actual equivalencing procedure that we describe in this section. We therefore assume throughout that $\mathbf{w}_{j}=\mathbf{0}$, and only reintroduce these inputs - exactly as in (7), (8) - when implementing the simulations for Section IV.

Now write $\mathbf{A}_{d}=\operatorname{Diag}\left\{\mathbf{A}_{j}\right\}$, and similarly define $\mathbf{B}_{d}, \mathbf{C}_{d}$ and $\mathbf{D}_{d}$. (The ordering of the subsystems in these blockdiagonal matrices is implied by the arrangement of variables in (10) below.) Combining (8) and (9) to eliminate $\mathbf{y}$, and letting $\mathbf{J}=\mathbf{D}_{\boldsymbol{d}}-\mathbf{Y}$, we obtain the DAE description

$$
\left[\begin{array}{c}
\dot{\mathbf{x}}_{r} \\
\mathbf{0} \\
\mathbf{0} \\
\dot{\mathbf{x}}_{z}
\end{array}\right]=\left[\begin{array}{cccc}
\mathbf{A}_{d r} & \mathbf{B}_{d r} & \mathbf{0} & \mathbf{0} \\
\mathbf{C}_{d r} & \mathbf{J}_{r r} & \mathbf{J}_{r z} & \mathbf{0} \\
\mathbf{0} & \mathbf{J}_{z r} & \mathbf{J}_{z z} & \mathbf{C}_{d z} \\
\mathbf{0} & \mathbf{0} & \mathbf{B}_{d z} & \mathbf{A}_{d z}
\end{array}\right]\left[\begin{array}{c}
\mathbf{x}_{r} \\
\mathbf{u}_{r} \\
\mathbf{u}_{z} \\
\mathbf{x}_{z}
\end{array}\right]
$$


Here we have separated the relevant $(r)$ and less relevant $(z)$ subsystems. The loads are treated as relevant subsystems and are retained intact (within $\mathbf{J}_{r r}$ ) during the equivalencing process. Subsequent to the equivalencing, any load buses that are amenable to a Ward-type reduction can be supressed. Note that elimination of the algebraic variables $\mathbf{u}_{r}, \mathbf{u}_{z}$ in (10) yields (2).

\section{A. Single-Eigenvalue Equivalent}

Now suppose we are interested in constructing a dynamic equivalent for the case where only the motion corresponding to the single eigenvalue $\lambda_{i}$ is excited in the system. Replacing $\dot{x}_{z}$ in (10) by $\lambda_{i} \mathbf{x}_{z}$ - the characteristic SMA substitution - sets us up to implicitly obtain a relationship of the form (4). The resulting description is then

$$
\left[\begin{array}{c}
\dot{\mathbf{x}}_{r} \\
\mathbf{0} \\
\mathbf{0} \\
\mathbf{0}
\end{array}\right]=\left[\begin{array}{cccc}
\mathbf{A}_{d r} & \mathbf{B}_{d r} & \mathbf{0} & \mathbf{0} \\
\mathbf{C}_{d r} & \mathbf{J}_{r r} & \mathbf{J}_{r z} & \mathbf{0} \\
\mathbf{0} & \mathbf{J}_{z r} & \mathbf{J}_{z z} & \mathbf{C}_{d z} \\
\mathbf{0} & \mathbf{0} & \mathbf{B}_{d z} & \mathbf{A}_{d z}-\lambda_{i} \mathbf{I}
\end{array}\right]\left[\begin{array}{c}
\mathbf{x}_{r} \\
\mathbf{u}_{r} \\
\mathbf{u}_{z} \\
\mathbf{x}_{z}
\end{array}\right]
$$

or equivalently (after using the algebraic equations to eliminate $\mathbf{x}_{z}$, assuming the requisite inverse exists)

$$
\left[\begin{array}{c}
\dot{\mathbf{x}}_{r} \\
\mathbf{0} \\
\mathbf{0}
\end{array}\right]=\left[\begin{array}{ccc}
\mathbf{A}_{d r} & \mathbf{B}_{d r} & \mathbf{0} \\
\mathbf{C}_{d r} & \mathbf{J}_{r r} & \mathbf{J}_{r z} \\
\mathbf{0} & \mathbf{J}_{z r} & \mathbf{J}_{z z}+\tilde{\mathbf{J}}_{z z}\left(\lambda_{i}\right)
\end{array}\right]\left[\begin{array}{c}
\mathbf{x}_{r} \\
\mathbf{u}_{r} \\
\mathbf{u}_{z}
\end{array}\right]
$$

where

$$
\tilde{\mathbf{J}}_{z z}\left(\lambda_{i}\right)=\mathbf{C}_{d z}\left(\lambda_{i} \mathbf{I}-\mathbf{A}_{d z}\right)^{-1} \mathbf{B}_{d z}
$$

Thus $\tilde{\mathbf{J}}_{z z}\left(\lambda_{i}\right)$ is the single-eigenvalue dynamic equivalent! The block-diagonal structure of $\tilde{\mathbf{J}}_{z z}\left(\lambda_{i}\right)$ implies that it takes the form of a non-standard, but easily implementable linear "admittance" load at each of the $z$-buses. This load is an incremental current injection whose phasor has real and imaginary parts that are linear combinations of the real and imaginary parts of the bus voltage perturbation phasor. The two required linear combinations are simply read off from $\tilde{\mathbf{J}}_{z z}\left(\lambda_{i}\right)$, although they are not as trivially related to each other as for a standard admittance load. The reduced-order model (12) now preserves the eigenvalue $\lambda_{i}$ of the full model (10).

\section{B. Multi-Mode Equivalent}

Consider a situation in which only the motion corresponding to the eigenvalue $\lambda_{i}$ is excited in the system. Then

$$
\mathbf{u}_{z}(t)=\mu_{z i} e^{\lambda_{i} t}
$$

where $\mu_{z i}$ is the vector corresponding to the $z$-bus voltages in the $i$ th (augmented) eigenvector of the DAE model (10) - i.e. $\mu_{z i}$ is the solution for $\mathbf{u}_{z}$ in (11) when $\mathbf{x}_{r}=\mathbf{v}_{r i}$, $\dot{\mathbf{x}}_{r}=\lambda_{i} \mathbf{v}_{r i}$, and $\mathbf{x}_{z}=\mathbf{v}_{z i}$, with $\mathbf{v}_{r i}$ and $\mathbf{v}_{z i}$ as defined following (5). From (14) we see that the $\lambda_{i}$-dependent term in (12) enters that equation via $\tilde{\mathbf{J}}_{z z}\left(\lambda_{i}\right) \mu_{z i}$. This suggests an SMA-style approach to obtaining a multi-mode equivalent: Find a constant matrix $\hat{\mathbf{J}}_{z z}$ such that

$$
\hat{\mathbf{J}}_{z z} \mu_{z i}=\tilde{\mathbf{J}}_{z z}\left(\lambda_{i}\right) \mu_{z i}
$$

for all $i$ corresponding to the equivalencing modes. For the typical case where there are many generators to be equivalenced, and only the few inter-area modes are used for equivalencing, there will be many solutions of (15), but it is certainly possible to have a unique solution or no solutions. When there is a solution $\hat{\mathbf{J}}_{z z}$ in (15), it constitutes a multi-mode equivalent, and is used in place of $\tilde{\mathbf{J}}_{z z}\left(\lambda_{i}\right)$ in (12). The resulting reduced-order system is thus

$$
\left[\begin{array}{c}
\dot{\mathbf{x}}_{r} \\
\mathbf{0} \\
\mathbf{0}
\end{array}\right]=\left[\begin{array}{ccc}
\mathbf{A}_{d r} & \mathbf{B}_{d r} & \mathbf{0} \\
\mathbf{C}_{d r} & \mathbf{J}_{r r} & \mathbf{J}_{r z} \\
\mathbf{0} & \mathbf{J}_{z r} & \mathbf{J}_{z z}+\hat{\mathbf{J}}_{z z}
\end{array}\right]\left[\begin{array}{c}
\mathbf{x}_{r} \\
\mathbf{u}_{r} \\
\mathbf{u}_{z}
\end{array}\right]
$$

and preserves the equivalencing modes of the full system. In general, a matrix $\hat{\mathbf{J}}_{z z}$ that satisfies (15) no longer has the block-diagonal structure that $\tilde{\mathbf{J}}_{z z}\left(\lambda_{i}\right)$ in (13) had, so it is not implementable via a new load at each of the $z$ buses separately. However, it is representable as a linear multi-port "admittance" presented across all the $z$-buses together. What is required now is a current injection at each $z$-bus that is a linear function of the voltages at all the $z$-buses. Again, the computation is simple.

When the solution of (15) is non-unique, there are many ways to proceed. For instance, note from (13) that $\tilde{\mathbf{J}}_{z z}\left(\lambda_{i}\right)$ in (15) has the constant matrix $\mathbf{C}_{d z}$ as its first term. We could thus look for solutions of (15) in the more restricted form

$$
\hat{\mathbf{J}}_{z z}=\mathbf{C}_{d z} \mathbf{L}
$$

with $\mathbf{L}$ now chosen to satisfy

$$
\mathbf{L}_{z z} \mu_{z i}=\left(\lambda_{i} \mathbf{I}-\mathbf{A}_{d z}\right)^{-1} \mathbf{B}_{d z} \mu_{z i}
$$

for all the equivalencing modes. This equation itself may have no solutions, a unique solution, or many solutions. For a $\hat{\mathbf{J}}_{z z}$ chosen according to (17) and (18), one can show that the reduced-order state-space model obtained by eliminating the algebraic variables from (16) is exactly of the form (3), (6), with a $\mathbf{K}$ that satisfies (5).

The above discussion is predicated on the availability of the requisite modal information, specifically $\lambda_{i}$ and $\mu_{z i}$ for all the equivalencing modes. With intermediate-sized models, this information can be obtained by full eigenanalysis of the model (2) or (10). However, for very large models, the task of obtaining the requisite information can itself be challenging. We defer further examination of this issue to future work too, merely noting here that efficient methods exist to compute selected modes (such as the inter-area modes that are of key interest to us) in very large systems, see for instance [16], [17].

\section{PERformance Evaluation}

We describe in this section experiments that we performed for one (slightly unstable) operating condition of the 93rd-order, 10-generator, 39-bus New England model [2] that has been used in a large variety of studies.

\section{A. Linear Models}

A 2-area partition constructed using $\sigma_{0}$ and $\sigma_{1}$ (the two slowest swing modes of the system) consists of (the very large equivalent) generator 10 in one area and the rest of the generators in the other area. A 3-area partition 


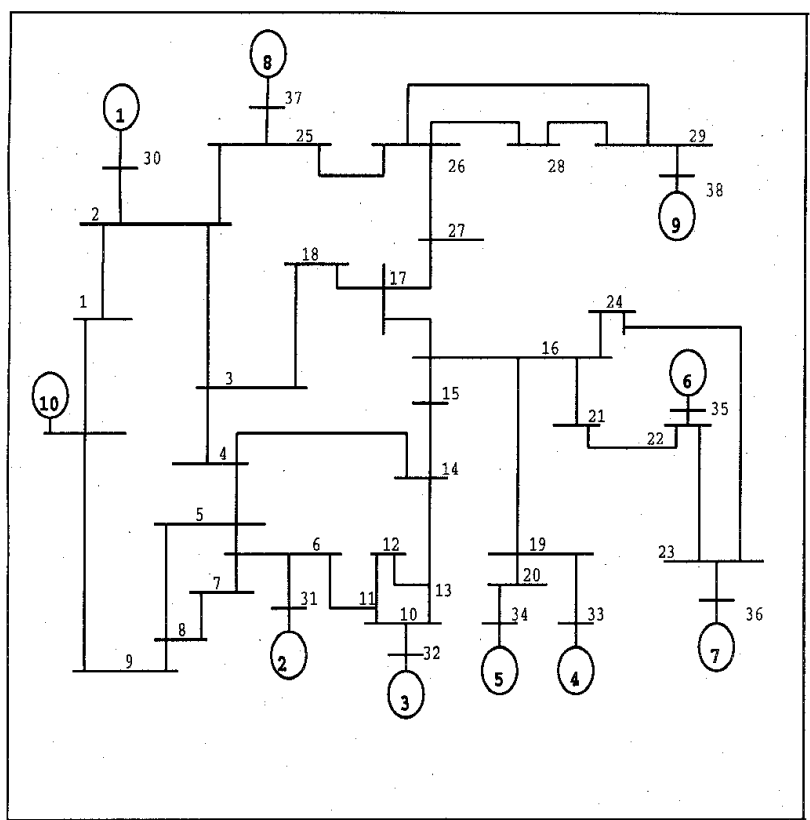

Figure 1: New England System Line Diagram. The synchronic areas were determined to be $\{6,4,5,7,9\}$ (which we selected as study area), $\{1,2,3,8\}$, and $\{10\}$. The numbers in boldface indicate reference generators.

using the same two modes yeilds a more interesting partition. Note that obtaining more areas than the number of modes in the chord is a privilege offered by our synchrony-based grouping algorithm, [13], [15], but not by the slow-coherency framework until recently, see [4].

The 3-area partition produced by our algorithm is specified in Figure 1. We selected the area consisting of generators $\{6,4,5,7,9\}$ as the study area; the two external areas are then $\{1,2,3,8\}$, and $\{10\}$. The reference generators are indicated in boldface. In addition to $\sigma_{0}$ and $\sigma_{1}$, the additional inter-area swing mode for this partition was determined by our chord selection procedure, [13], [15], to be $\sigma_{3}$ (and not $\sigma_{2}$, which is the next-slowest mode). Due to the nontrivial choice of inter-area modes involved, as well as to synchrony-based grouping and reference generator selection, the 3-area partition in Figure 1 is significantly different from what is obtained by coherency-based methods. The algorithm of [3], applied to the chord comprising $\sigma_{0}, \sigma_{1}$, and $\sigma_{2}$, produces the areas $\{\mathbf{5}, 2,3,4,6,7\},\{\mathbf{9}, 8\}$, and $\{10,1\}$, while the slightly modified algorithm in [16] produces $\{\mathbf{5}, 2,3,4,6,7\},\{\mathbf{9}$, $8,1\}$, and $\{10\}$. We shall see that the synchrony-based partitioning in this case, despite its completely different way of decomposing the system, is able to demarcate a study area in a strikingly effective way.

The equivalent used in the following experiments was constructed according to the prescription in Section III.B. The equivalencing modes were chosen to be just the interarea electromechanical modes, namely the non-oscillatory modes associated with the two real eigenvalues of the detailed model that correspond to $\sigma_{0}$, plus the oscillatory
Table 1: Preservation of Relevant Electromechanical Modes. The inter-area modes were used in the equivalencing, and so are preserved exactly in the reduced model. However, the remaining intra-area modes of the study area are preserved very well too, including the one unstable pair.

\begin{tabular}{||l|r|r||}
\hline & Full System & Reduced System \\
\hline Inter-Area & $0.0000+j 0.0000$ & $0.0000+j 0.0000$ \\
Modes & $-0.1011+j 0.0000$ & $-0.1011+j 0.0000$ \\
& $-0.2954 \pm j 3.8402$ & $-0.2954 \pm j 3.8402$ \\
& $-0.2857 \pm j 7.0972$ & $-0.2857 \pm j 7.0952$ \\
\hline Intra-Area & $-0.2660 \pm j 6.0316$ & $-0.2849 \pm j 6.0969$ \\
Modes & $0.0018 \pm j 6.9580$ & $0.0264 \pm j 6.9223$ \\
& $-0.3445 \pm j 8.6709$ & $-0.3430 \pm j 8.7186$ \\
& $-0.4114 \pm j 9.0271$ & $-0.4105 \pm j 9.0205$ \\
\hline
\end{tabular}

modes associated with the two complex pairs of eigenvalues that correspond to $\sigma_{1}$ and $\sigma_{3}$. Since there are three generators to be equivalenced (namely 2,3 , and 8 ), the solution for $\hat{\mathbf{J}}_{z z}$ in (15) is unique.

The electromechanical modes of the reduced system and the corresponding relevant modes of the original system are shown in Table 1. Note that even though the intraarea modes were not used in the equivalencing, these modes - including the one unstable pair - are preserved well. The remaining (non-electromechanical) intra-area modes are not listed, but are preserved at least as well as the electromechanical modes in Table 1.

Evaluating the performance of the reduced system in the linear case can be done effectively by looking at the frequency response. Figure 2 shows an example of Bode plots that compare the frequency responses of the full and reduced models, with exceptionally good match. Error plots are also given, for the reduced system (16), and for the reduced system without the equivalent, i.e. with $\hat{\mathbf{J}}_{z z}=\mathbf{0}$ in (16), or equivalently with $\hat{\mathcal{A}}=\mathcal{A}_{r r}$ in (6). Notice how, in the frequency range of primary interest (1-10 $\mathrm{rad} / \mathrm{s}$ ), the performance of the reduced system without the equivalent is only slightly inferior to that of the reduced system with the equivalent.

What is happening here is that, although we picked generator 4 rather arbitrarily as the one to apply disturbances to, these disturbances turn out to excite primarily the intra-area modes. The modal equivalencing, which is focused on the inter-area modes, therefore does not play a major role in this case (but will become more apparent with some of the results for the nonlinear simulations described in the next subsection). It is striking that synchrony produces such a crisp definition of areas.

\section{B. Nonlinear Models}

Although our dynamic equivalent is constructed using a linearized theory, it is certainly possible to embed the resulting equivalent in a model that represents nonlinearities in the study-area generators and/or nonlinearities in the coupling of the study area to the rest of the system. This is what we do next. 'The time-domain simulations 

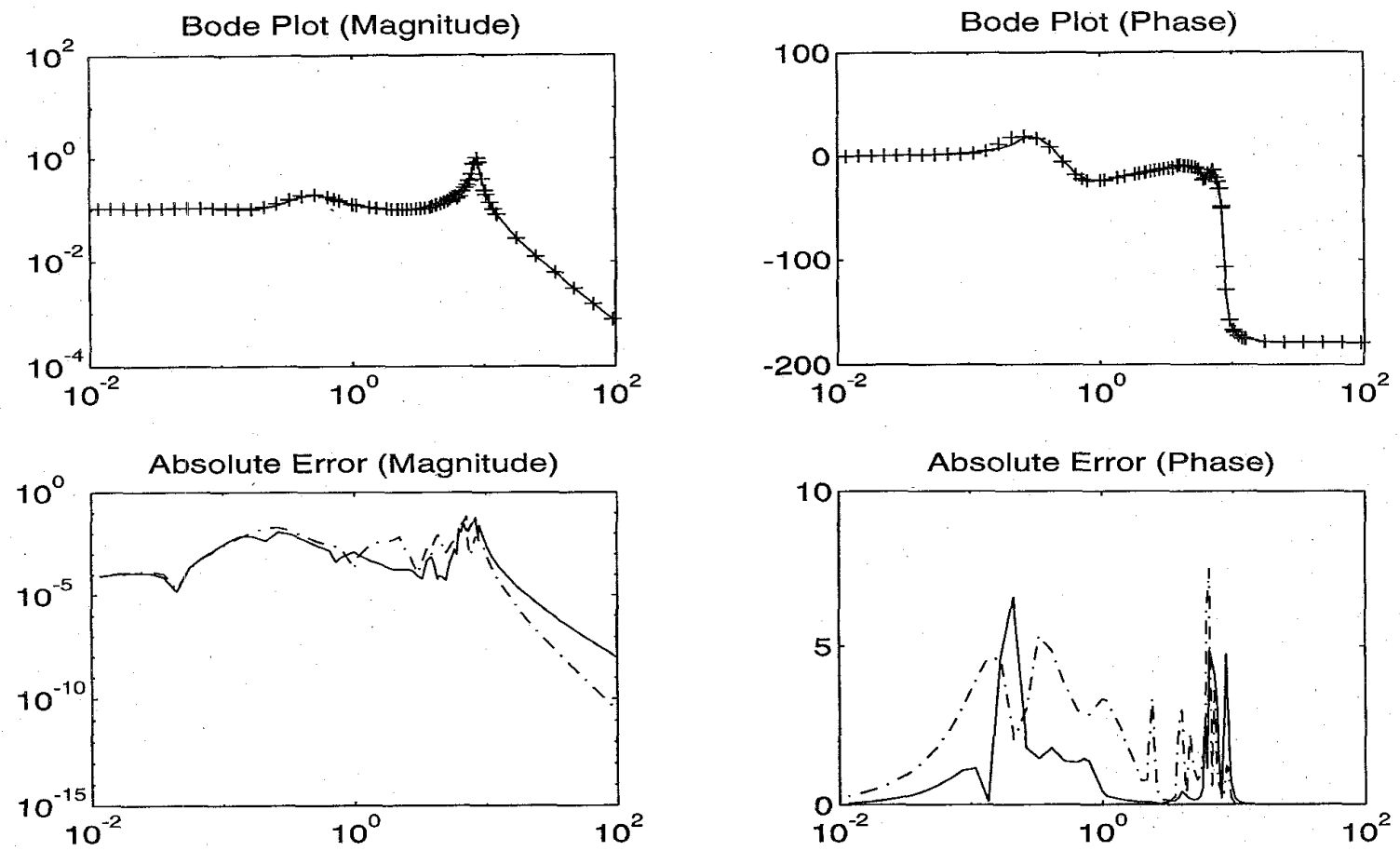

Figure 2: Bode Plots for the Full and Reduced Linear Systems. The input is the mechanical power perturbation at generator 4, and the output is the angle of generator 4, measured with respect to the angle of generator 6 (reference generator). The top two figures show the magnitude and phase for the Bode plot, respectively, for the full system $(-)$ and the reduced system with the equivalent (+). In the bottom two figures the solid (-) plots show the absolute errors for the top two figures, and the dotted (-.) plots show the corresponding absolute errors for the reduced system without the equivalent (i.e. $\hat{\mathbf{J}}_{z z}=\mathbf{0}$ ). The horizontal axis corresponds to the frequency measured in radians/s. The phase is indicated in degrees.

were run with the EUROSTAG package, [18], [1], which uses a variable-time-step integration routine.

We performed a number of experiments in which we simulated the full and reduced systems. The equivalent computed using (15) was easily implemented in the reduced model, using the voltage-controlled current injectors available within the software. The vector $\eta_{z}$ of injected currents at the $z$-buses is given by

$$
\boldsymbol{\eta}_{\boldsymbol{z}}=\mathbf{I}_{0}+\hat{\mathbf{J}}_{z z} \mathbf{u}_{z}
$$

where $\mathbf{I}_{0}$ is a vector of currents (automatically computed by the program) that correspond to the nominal steadystate outputs of the generators being replaced. This equation is directly coded via a block-diagram entry.

Figure 3 shows results obtained for perturbations at generator 4. As before, results for the full model are compared with those for two different reduced models, one without any equivalent $\left(\hat{\mathbf{J}}_{z z}=0\right)$ and one with the equivalent. For the mechanical perturbation, both reduced models perform exceptionally well. The reduced system with the equivalent is only slightly better than the one without the equivalent, suggesting that: (i) the synchrony-based definition of areas has produced very well defined areas; and (ii) the particular disturbance we used excites primarily the intra-area modes. Note that the unstable mode in Table 1 seems to be not significantly excited in these experiments.
For the perturbation of the voltage set point of the AVR, Figure 3 shows that the modal equivalencing step makes a significant difference. The reduced model with no equivalent performs reasonably at first, but then breaks out into large spurious oscillations. Inclusion of the modal equivalent produces a reduced model that matches excellently the response of the full model.

Our limited studies of this test example have served to confirm the important role that is played by the two main ingredients of our dynamic equivalencing approach: synchronic area determination, and modal equivalencing. However, a fuller evaluation (including tests with more severe disturbances, and comparisons with other approaches) has to be left to future work.

\section{CONCLUSIONS}

The work reported here opens up a new avenue for dynamic equivalencing, and raises several questions for further investigation. We list some of these questions here.

- The weak-interconnections model yields localizability in the slow-coherency framework; we do not yet have a corresponding benchmark model for synchrony. However, numerical experience so far suggests that synchrony reveals localizability very effectively. 

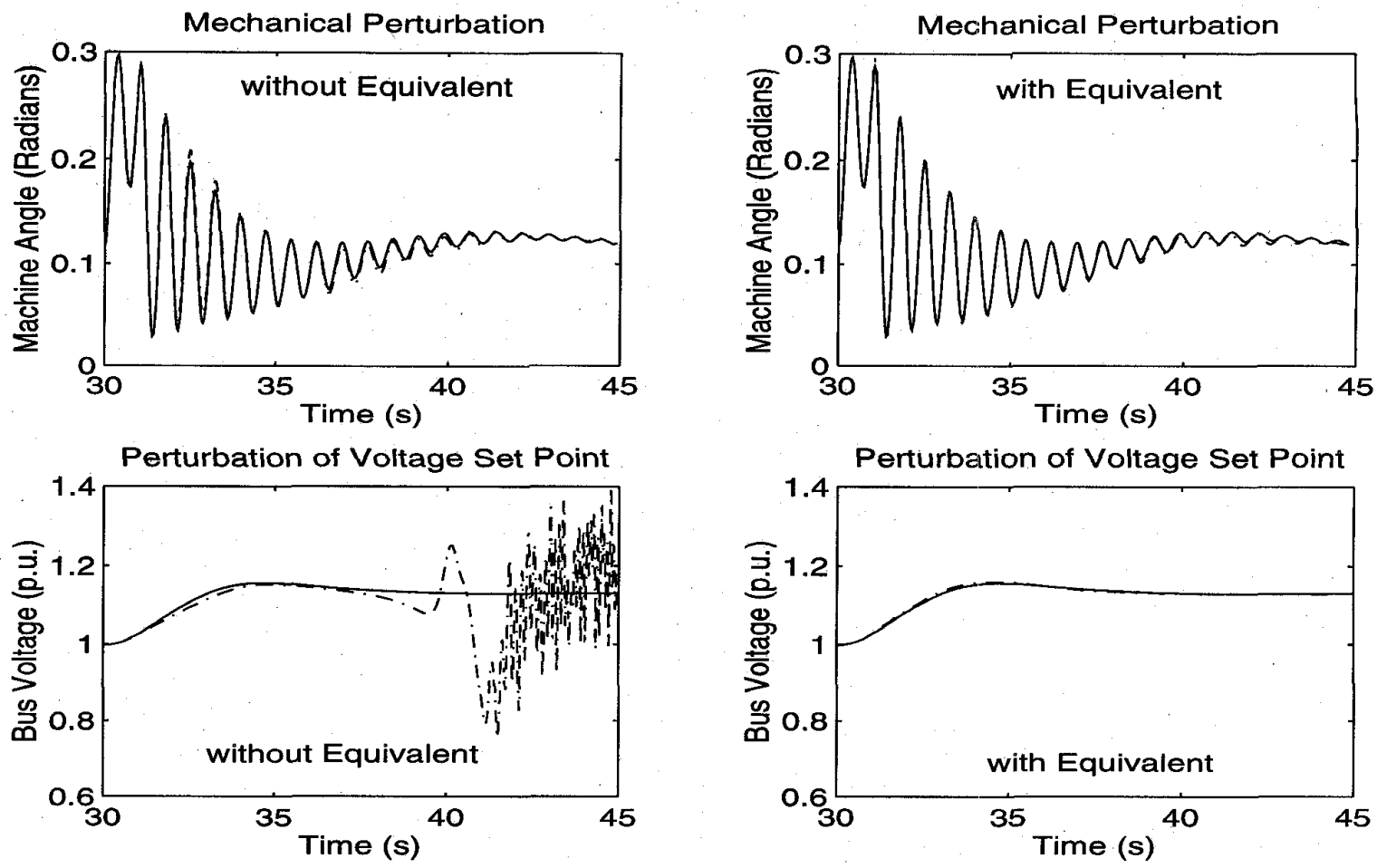

Figure 3: Nonlinear Simulation using EUROSTAG. The top two figures show the response of the full system (-) and the reduced system (-.), without and with the equivalent, for a 1 p.u. mechanical perturbation of duration $1.0 \mathrm{~s}$ (60 cycles) applied at generator 4 . The response shown is the angle (in radians) at generator 4 with respect to the angle at generator 6 (the reference generator for the study area). The bottorn two figures show the response of the full system (-) and the reduced system (-.), without and with the equivalent, when the voltage set point of the AVR of generator 4 was perturbed from 0.97 (p.u.) to 1.1 (p.u.). The response shown is the voltage at the bus of generator 4 . In both plots the perturbations were introduced at time $t=30 s$.

- Careful study is needed of the relative practical merits of equivalencing with all relevant modes, versus with just the inter-area modes. If only inter-area modes are retained, one has the challenge of using the available degrees of freedom to avoid perturbing the intra-area modes significantly.

- The computational burden needs to be carefully assessed, particularly for large models. Efficient methods for the analysis of selected modes in such large models, [16], [17], should be incorporated.

- Analytical error bounds for the performance of our dynamic equivalents would be valuable.

- A very important challenge is that of directly identifying dynamic equivalents in the structure presented here. The flavor of what we have in mind can be seen in the very preliminary explorations in [22].

\section{ACKNOWLEDGMENTS}

This paper has grown out of a project at the Laboratory for Electromagnetic and Electronic Systems, MIT, funded by Electricité de France (EDF). The authors are grateful to Dr. Bruno Meyer and Dr. Christine Vialas of EDF, who initiated and nurtured the project. G. N. Ramaswamy, L. Rouco, and G. C. Verghese are also grateful for partial support from the Instituto de
Investigación Tecnológica, Madrid. Additional support for G. C. Verghese came through a sabbatical grant from MIT and a grant from the Dirección General de Investigación Científica y Técnica of the Spanish Ministry of Education and Science.

\section{REFERENCES}

[1] Astic, J. Y., Bihain, A., and Jerosolimski, M., , "The Mixed Adams - BDF Variable Step Size Algorithm to Simulate Transient and Long Term Phenomena in Power Systems," IEEE Transactions on Power Systems, Vol. 9, No. 2, pp. 929-935, May 1994.

[2] ByERLY, R. T., Sherman, D. E., and BENNON, R. J., "Frequency Domain Analysis of Low-Frequency Oscillations in Large Electric Power Systems," EPRI Report EL-727 on Research Project RP 744-1 carried out by Westinghouse Electric Corporation, April 1979 .

[3] CHOw, J. H., Time-Scale Modeling of Dynamic Networks with Applications to Power Systems, Lecture Notes in Control and Information Sciences, Vol. 46, Springer Verlag, 1982.

[4] Chow, J. H., and Cheung, K. W., "A Toolbox for Power System Dynamics and Control Engineering Education and Research," IEEE Transactions on Power Systems, Vol. 7, No. 4, pp. 1559-1564, November 1992.

[5] Date, R. A. and ChOW, J. H., "Aggregation Properties of Linearized Two-Time-Scale Power Networks," IEEE Transactions on Circuits and Systems, Vol. 38, No. 7, pp. 720-730, July 1991.

[6] De Oliveira, S. E. M., De Queiroz, J. F., and Massaud, A. G., "Modal Dynamic Equivalent for Electric Power Systems: Parts 
I and II," IEEE Transactions on Power Systems, Vol. 3, No. 4, pp. 1723 - 1737, November 1988.

[7] Eliasson, B. E., and LINDAHL, S. O. R.,, "A New Coherence Approach of Generators for Investigation of Slow and System Wide Oscillations in Large Power Systems," IFAC Symposium on Power Systems and Power Plant Control, Seoul, Korea, 1989.

[8] Gezves, S., "A Modal Coherency Technique for Deriving Dynamic Equivalents," IEEE Transactions on Power Systems, Vol. 3, pp. 44-51, February 1988.

[9] Germond, A. J. and Podmore, R., "Dynamic Aggregation of Generating Unit Models," IEEE Transaction on Power Apparatus and Systems, Vol. PAS-97, No. 4, pp. 1060-1069, July/August 1978.

[10] Kundur, P., Morison, G. K., Bal.u, N. J., and Lauby, M.G., "Advanced Software for Power System Analysis," Workshop on New Issues in Power System Simulation, International Electric Research Exchange, pp.20-29, Caen, France, March 1992.

[11] Pérez-Arriaga, I. J., Verghese, G. C., Pagola, F. L., Sancha, J. L., and SchwEFPE, F. C., "Developments in Selective Modal Analysis of Small-signal Stability in Electric Power Systems," Automatica, Vol. 26, No. 2, pp. 215-231, 1990.

[12] PODMORE, R., "Identification of Coherent Generators for Dynamic Equivalents," IEEE Transactions on Power Apparatus and Systems, Vol. PAS-97, No. 4, pp. 1344-1354, July/August 1978.

[13] Ramaswamy, G. N., Verghese; G. C., Vialas, C., and DeMarco, C. L., "Going Beyond Coherency: Synchrony as a Basis for Dynamic Equivalencing in Power System Models," North American Power Symposium, Howard University, Washington, D. C., October 1993.

[14] Ramaswamy, G. N., Verghese, G. C., Rouco, L., Lesieutre, B. C., and FILIÁTEE, O., "Synchronic Modal Equivalencing (SME): A New Framework for Constructing Dynamic Equivalents in Power Systems," North American Power Symposium, Kansas State University, Manhattan, Kansas, September 1994.

[15] Ramaswamy, G. N., Verghese, G. C., Rouco, L., Vial.as, C., and DEMARCo, C. L., "Synchrony, Aggregation and Multi-Area Eigenanalysis, IEEE PES Winter Meeting, New York, January 1995.

[16] Rouco, L. and PEREz-ARRIAGA, I. J., "Multi-area Analysis of Small Signal Stability in Large Electric Power Systems by SMA," IEEE Transactions on Power Systems, Vol. 8, No. 3, pp. 1257-1265, August 1993.

[17] Rouco, L., Pérez-Arriaga, I. J., Crlado, R., and Soto, J., "A Computer Program Package for Analysis of Small Signal Stability in Large Electric Power Systems," Proceedings of the 11th Power Systems Computation Conference, pp. 1141-1148. Avignon (France), September 1993.

[18] Stubbe, M., Bihain, A., Deuse, J., and BaAder, J. C., "STAG: A New Unified Software Program for the Study of the Dynamic Behaviour of Electrical Power Systems," IEEE Transactions on Power Systems, Vol. 4, No. 1, pp. 129-138, February 1989.

[19] Troullinos, G. and Dorsey, J.F., "Coherency and Model Reduction: A State-Space Point of View," IEEE Transactions on Power Systems, Vol. 4, No. 3, pp. 988 - 995, August 1989.
[20] UNDRILL, J. M. and TURNer, A. E., "Construction of Power Systems Electromechanical Equivalents by Modal Analysis," IEEE Transactions on Power Apparatus and Systems, Vol. PAS-90, pp. 2049-2059, September/October 1971.

[21] VAN OrRsouw, P.M., "A Dynamic Equivalent Using Modal Coherency and Frequency Response," IEEE Transactions on Power Systems, Vol. 5, No. 1, pp. 289 - 295, February 1990.

[22] Vialas, C., Verghese, G. C., Rejes, J. N., and Ramaswamy, G. N., "Prony Identification of Modal Dynamic Equivalents," North American Power Symposium, Kansas State University, Manhattan, Kansas, September 1994.

[23] Zaborsky, J., Whang, K.-W., HuANG, G.M.; Chiang, L.-J., and LIN, S.-Y., "A Clustered Dynamical Model for a Class of Linear Autonomous Systems Using Simple Enumerative Sorting," IEEE Transaction on Circuits and Systems, Vol. CAS-29, No. 11, pp. 747-757, November 1982 .

\section{BIOGRAPHIES}

Ganesh N. Ramaswamy (S'90) was born in Kuala Lumpur, Malaysia He received his Bachelor of Science and Master of Science degrees in Electrical Science and Engineering from MIT in 1992, and is currently pursuing his doctoral studies, also at MIT. His research interests include modeling and identification of large dynamic systems, model-based signal processing, and communication systems. He is a member of Tau Beta Pi, Eta Kappa Nu and Sigma Xi.

Luis Rouco (S '89, M '91) received the Ingeniero Industrial and Doctor Ingeniero Industrial degrees from the Universidad Politécnica de Madrid in 1985 and 1990, respectively. He is currently a Research Associate at the Instituto de Investigación Tecnológica and a faculty member at the School of Engineering of the Universidad Pontificia Comillas.

Ollivier Fillâtre received his engineering and doctoral degrees from the Ecole Centrale de Paris in 1988 and 1991 respectively, with a specialization in numerical analysis from Paris VI University in 1988 . He has been with Electricité de France (EDF) since 1992, working with EDF"s $R$ \& D Division on power system simulation.

George C. Verghese (S '74, M '78) received his B.Tech. from IIT, Madras, M.S. from SUNY, Stony Brook, and Ph.D. from Stanford University. He joined the EECS Department at MIT in 1979, where he is Professor of Electrical Engineering, attached to the Laboratory for Electromagnetic and Electronic Systems. He has served as Associate Editor of Automatica and of the IEEE Trans. Automatic Control, and on the AdCom of the IEEE Power Electronics Society. He is coauthor of Principles of Power Electronics, Addison-Wesley, 1991.

Patrick Panciatici received his engineering degree from the Ecole Supérieure d'Electricité in 1984. He joined EDF's R \& D Division in 1985 and where he is presently the EUROSTAG team leader. His main interests are power system simulation and scientific computation.

Bernard C. Lesieutre (S '86, M '93) received his B.S., M.S., and Ph.D.degrees in electrical engineering from the University of Illinois at Urbana-Champaign in 1986, 1988, and 1993 respectively $\mathrm{He}$ is currently an Assistant Professor of Electrical Engineering at the Massachusetts Institute of Technology. His research interests include machine modeling and power system dynamics, stability and control. $\mathrm{He}$ is a member of Eta Kappa Nu.

David Peltier is with EDF as a Mastère student of the Ecole Cen trale de Paris. He received his engineering degree from the Institut en Sciences et Technologie, Paris VI University, in 1993. 


\section{Discussion}

M. K. Pal (Consultant, Edison, NJ): The authors have presented a methodology for forming dynamic equivalents in the study of large interconnected power systems. We have several comments on the authors' approach and would like the authors to respond.

The derivation in Section III suggests that in the equivalencing process the external generators, except for the reference ones, are replaced by injected currents at the generator buses. Statements to this effect are also made in the paper. The authors also state that the loads are treated as relevant subsystems and are retained intact during the equivalencing process. There is therefore no reduction in the system size in terms of the number of buses. Only the differential equations corresponding to the equivalenced generators are being eliminated. In a dynamic simulation, a major part of the computation is taken up by the network solution. So, even if the equivalent works well, the reduction in solution time would be quite modest. If the loads are passive, and the load buses are eliminated subsequent to the equivalencing, in the conventional way, as stated by the authors, the network sparsity will be lost and the solution time might increase further.

The argument presented for choosing one reference generator from each external area is not quite clear to us. We agree that a knowledge of the motion of a single generator in a synchronic group would be enough to know the motion of the remaining generators in the group. However, this would not be true if the other generators are eliminated in the way described by the authors. Also note that the inter-area modes are determined by the combined inertia of the machines in each area as well as the tie line impedances. We note that the inertias of the equivalenced machines enter into $\hat{\mathrm{J}}_{z z}$, and the equivalent is implemented using the voltage controlled current injection as shown in equation (19). However, we do not think this will account for the effect of inertia correctly. This is because the voltages at these (z) buses will hardly change. The effect of inertia can be correctly accounted for only through swing equations. Inertias of machines away from the study area will not play a dominant role in conventional transient and small-signal stability analyses. However, in studies of interarea oscillations and long-term simulations in large interconnected systems correct representation of the total inertia in each area is important.

We think one could easily improve on the authors' equivalent by simply representing the generators outside the study area by the classical swing equation model, as long as these generators are electrically remote from the study area. Although this will add $2 \mathrm{n}$ state variables to the system description, where $n$ is the number of generators intended for equivalencing, the overall computing time may be lower as compared to that required by the authors' methodology. The problems associated with using equivalents derived from a linearized analysis in studies involving nonlinearities will also be eliminated. This is, of course, not a substitute for a good equivalent, since it does not reduce the system size, and therefore the computational burden significantly.

\section{P. Kundur, L. Wang, and G.K. Morison, Powertech} Labs Inc, Surrey, British Columbia, Canada : The authors are to be congratulated on this interesting paper which opens up a new way for constructing dynamic equivalents of power systems. The idea of using a linear multi-port "admittance" is computationally attractive for drastically reducing the system dynamics. Another interesting feature of the proposed method is that the modal properties associated with the study area are preserved in the reduced model which is therefore suitable for small-signal stability studies.

We have several questions related to the application of this method:

1. The equivalents of the external irrelevant generators are expressed as a linear multi-port "admittance". The validity of using such a model for transient stability studies is to be proven, since inherently the system behaviour following large disturbances is affected by the non-linearities of the system. Would the authors comment on how the proposed linear equivalents can handle such nonlinear characteristics?

2. When performing dynamic reductions, it is desirable to reduce both the generators and network in order to achieve maximum computational efficiency. However, it appears that the proposed approach requires that all generator buses be fully retained so that voltages at these buses can be calculated and the equivalent current injections applied when working with the reduced system model. Does this imply that only the dynamic equations representing the generating units can be reduced while the entire network has to be kept?

3. Regarding the numerical examples given in the paper, Figures 2 and 3 compare the results of two different reduced models, one with the equivalent and the other without. The paper indicates that the model without an equivalent was obtained by simply setting the matrix $\mathbf{J}_{z z}=\mathbf{0}$. From Equation 16, this appears to imply that netting of a generator is achieved by simply inserting its source admittance (the terms in matrix $\mathbf{D}_{j}$ of Equation 8) into the network admittance matrix. Does this mean that for time-domain analysis, a netted unit appears as a short circuit through the machine transient impedance? Could the authors clarify?

The authors have reported on experiments using the 39bus New England test case. Naturally, the main interest by utilities is in the dynamic reductions of large systems. Reductions on systems with 12,000 buses and 2,000 machines with full controls are not uncommon, and even larger system capabilities are being pursued by utilities in North America. We look forward to the evaluation (through both eigenvalue and time-domain analyses) of the author's technique on large practical systems.

Manuscript received February 17, 1995. 
George C. Verghese, Ganesh N. Ramaswamy, Ollivier Fillatre, and Bernard C. Lesieutre: We thank the discussers for their careful reading of our paper. As the approach to dynamic equivalencing that is described in the paper is under continuing development and extension, their comments and questions are particularly useful.

We respond first to the remarks and questions of Drs. Kundur, Wang and Morison. We agree completely that considerably more testing, as well as extension to much larger models, will be required to establish SME as a practically significant route to dynamic equivalencing. The paper lays out a preliminary framework and presents the results of some initial exploratory tests, but much more remains to be done. The extension to very large models, for instance, will require folding in approaches of the sort described in [15], [16], [17]. Some generalizations of the basic framework have been obtained since the paper was prepared, and are contained in [A].

Question 1 asks about the retention of nonlinear characteristics. The system behavior after a large disturbance is undoubtedly affected by nonlinearities of the system. It is important to note that our equivalent comprises not only the linear multiport "admittance" that is the focus of the discussers' question, but also the external reference generators, one for each external synchronic group. These reference generators, along with all the generators in the study group, may be represented in full nonlinear detail, thus providing a direct mechanism for including nonlinear effects in the equivalenced system.

As for the multiport "admittance", our limited experience so far (see also the clarifications below in response to Question 3) suggests that this "admittance" may often only provide a slight improvement over the equivalent constructed using zero "admittance", provided the synchronic grouping and selection of reference generators have been appropriately carried out. Thus, our synchronic grouping and reference generator selection procedures should be seen as essential ingredients of the proposed approach to equivalencing. (While our paper has used a fault-independent approach to selecting the synchronic groups, there are natural ways to consider carrying out fault-dependent synchronic grouping, with the chord $\nu$ being dependent on the fault. We are beginning to explore this possibility.)

Furthermore, our expectation is that the multiport "admittance" will continue to represent valid inter-relationships under large disturbances, as long as the synchronic structure recognized in a simple linearized swing-equation model is approximately retained for large disturbances in a full nonlinear model. The results in [12] suggest that any coherency structure which is recognized in the linearized swing model is approximately retained in the full nonlinear model, so one may hope that this is the case for synchrony as well.

As a final point in response Question 1, it should be noted that, as far as we are aware, all existing approaches to $\mathrm{dy}$ namic equivalencing with detailed (as opposed to swing equation) models end up with a rather $a d$ hoc approach to including nonlinearities in the equivalent. Our combination of nonlinear reference generators and a linear multiport "admittance", all chosen with some justification, would seem to be as reasonable as any other existing approach. In any case, further tests, with larger disturbances than those described in the paper, are certainly called for, and we intend to pursue these.
Question 2 asks what reductions of the network are possible. We agree that it is desirable to reduce not only the generator dynamics but also the network size, in order to achieve computational efficiency. Our paper failed to mention that a significant portion of the network can indeed be eliminated, without any increased penalty in loss of sparsity. Specifically, there is a maximal set of load buses that are connected only amongst themselves and to the equivalenced (i.e. replaced) generators, but to nothing else. These can be eliminated with the standard Ward-type reduction, the only effect being to modify the multiport "admittance", which in general was a full matrix to begin with. (The generalizations in [A] that were referred to earlier may offer some additional possibilities, but these remain to be explored.) Finally, of course, additional load buses can be eliminated in the standard way, but with a loss of sparsity.

Question 3 concerns the results in Figures 2 and 3, and the meaning of the "without equivalent" case. We are grateful to the discussers for drawing our attention to certain issues that were inconsistently dealt with in the paper, and to an associated omission, which we take this opportunity to correct. Recall first that the current injections into the network at the equivalenced generator buses (the $z$-buses) are represented as follows in our linearized model: we attach to the $z$-buses the computed multiport "admittance" $\widehat{\mathbf{J}}_{z z}$ in parallel with the (block-) diagonal admittance $\mathbf{D}_{d z}$, whose entries are the feedforward terms $\mathbf{D}_{j}$ from equation (8) for values of the index $j$ that correspond to the $z$-buses.

The "without equivalent" case shown in Figure 2 was obtained by setting $\widehat{\mathbf{J}}_{z z}=0$, and leaving only the term $\mathbf{D}_{d z}$. (It would perhaps have been better for us to give this case a different label, because our label suggests that the equivalent is fully embodied in $\widehat{\mathbf{J}}_{z z}$ alone, which is false - see the response to Question 1.) If we assume a classical synchronous machine model, this no-equivalent case in the linearized model will simply correspond to connecting the transient reactance between the equivalenced generator bus and ground, as the discussers note.

The "without equivalent" case in the nonlinear EUROSTAG simulation shown in Figure 3 was actually obtained by removing each of the equivalenced generators entirely and replacing them by just their nominal current injections, contained in the. vector $\mathrm{I}_{0}$ of (19). This corresponds to setting both $\widehat{\mathbf{J}}_{z z}$ and $\mathbf{D}_{d z}$ to zero, which is different from what was done for the "without equivalent" case in Figure 2.

We have subsequently repeated the nonlinear EUROSTAG simulations for the case where $\widehat{\mathbf{J}}_{z z}=0$ but the admittance $\mathbf{D}_{d z}$ is retained, to supplement the nominal current injections $\mathbf{I}_{0}$ at the $z$-buses. (In the case of a classical model, the retention of the term $\mathbf{D}_{d x}$ corresponds to placing the transient reactance between the equivalenced generator bus and a voltage source of value equal to the nominal bus voltage.) With this modification, the "without equivalent" result in the lower left plot of Figure 3 improves significantly, to where it is only marginally worse than the "with equivalent" case. The other three plots in Figure 3 are essentially unchanged by the modification. (We are grateful to Mr. Christophe Evrard of EDF for running these and related verifications in support of this closure.) What this reinforces is the major role played by the synchronic grouping and selection of reference machines the subsequent addition of $\widehat{\mathbf{J}}_{z z}$ to $\mathbf{D}_{d z}$ makes only a marginal improvement, at least in our limited tests so far. 
Finally, the omission that was mentioned earlier occurs in (19). The correct equation for the vector $\eta_{z}$ of injected currents at the $z$-buses should be written as

$$
\eta_{z}=\mathbf{I}_{0}+\left(\widehat{\mathbf{J}}_{z z}+\mathbf{D}_{d z}\right) \mathbf{u}_{z z}
$$

The "with equivalent" plots in Figure 3 are consistent with this equation.

We turn now to the comments of Dr. Pal. Our response to his first paragraph of comments, regarding the important issue of reducing the network itself, is contained in the response to Question 2 of Drs. Kundur et al. Our responses to Questions 1 and 2 above also address (as far as we are able) the points raised in his last paragraph of comments, where he proposes an alternative equivalencing scenario that he claims would "easily improve" on our equivalent. Careful studies will be needed to make the required comparisons and establish or disprove such claims; we do intend to carry out comparisons of the sort that Dr. Pal suggests. We should emphasize again that proper determination of the study area via synchronic grouping is itself a critical part of our framework - we are not clear how Dr. Pal proposes to delineate a study area for his approach, if he is indeed dismissing ours.

His second paragraph of comments asks about the rationale for choosing one reference generator for each external area. Our motivation is similar to that used in the case of coherency. However, he is right in suggesting (unless we have misunderstood him) that we make no direct use of the computed synchronic relationships beyond the grouping stage. Rather, after selecting the appropriate chord of inter-area modes and completing the synchronic grouping, we focus on obtaining a reduction that retains the equivalencing modes, via equation (15). By the same token, we cannot agree with Dr. Pal's comment that our approach is unable to properly account for the inertias of the replaced machines and specifically their effect on inter-area oscillations. Our approach is built exactly around retaining these modes in the equivalenced system. Srnall perturbations in the $z$-bus voltages are compensated for by correspondingly large gains embodied in $\widehat{\mathbf{J}}_{z z}$. An approach that stays closer to the synchronic relationships might be obtained by making heavier use of equation (5), and generalizations in this direction are in fact contained in [A]. These generalizations still involve current injections at the buses of the equivalenced generators, but the injections are dependent on variables in the external reference generators, and not on the voltages at the equivalenced buses.

In conclusion, we are very grateful to the discussers for their thoughtful and stimulating comments and questions on our paper.

\section{REFERENCE}

[A] Ramaswamy, G. N., "Modal Structures and Model Reduction, with Application to Power System Equivalencing," $\mathrm{Ph} . \mathrm{D}$. Thesis, Massachusetts Institute of Technology, June 1995.

Manuscript received April 17, 1995. 